\title{
The use of micro-costing in economic analyses of surgical interventions: a systematic review
}

Shelley Potter ${ }^{1,2 \dagger}$, Charlotte Davies ${ }^{1 *} \mathbb{B}$, Gareth Davies ${ }^{1}$, Caoimhe Rice ${ }^{3}$ and William Hollingworth ${ }^{3}$

\begin{abstract}
Background: Compared with conventional top down costing, micro-costing may provide a more accurate method of resource-use assessment in economic analyses of surgical interventions, but little is known about its current use. The aim of this study was to systematically-review the use of micro-costing in surgery.

Methods: Comprehensive searches identified complete papers, published in English reporting micro-costing of surgical interventions up to and including 22nd June 2018. Studies were critically appraised using a modified version of the Consensus on Health Economic Criteria (CHEC) Checklist. Study demographics and details of resources identified; methods for measuring and valuing identified resources and any cost-drivers identified in each study were summarised.
\end{abstract}

Results: A total of 85 papers were identified. Included studies were mainly observational comparative studies $(n=42,49.4 \%)$ with few conducted in the context of a randomised trial $(n=5,5.9 \%)$. The majority of studies were single-centre $(n=66,77.6 \%)$ and almost half $(n=40,47.1 \%)$ collected data retrospectively. Only half $(n=46,54.1 \%)$ self-identified as being 'micro-costing' studies. Rationale for the use of micro-costing was most commonly to compare procedures/techniques/processes but over a third were conducted specifically to accurately assess costs and/or identify cost-drivers. The most commonly included resources were personnel costs $(n=76,89.4 \%)$; materials/disposables $(n=76,89.4 \%)$ and operating-room costs $(n=62,72.9 \%)$. No single resource was included in all studies. Most studies $(n=72,84.7 \%)$ identified key cost-drivers for their interventions.

Conclusions: There is lack of consistency regarding the current use of micro-costing in surgery. Standardising terminology and focusing on identifying and accurately costing key cost-drivers may improve the quality and value of micro-costing in future studies.

Trial registration: PROSPERO registration CRD42018099604.

Keywords: Micro-costing, Economic evaluation, Surgery, Systematic review

\footnotetext{
* Correspondence: Charlotte.Davies@bristol.ac.uk

Precis: The use of micro-costing in economic analyses of surgery lacks

standardization. Focusing on cost-drivers may improve the quality and value of micro-costing in future studies.

tShelley Potter and Charlotte Davies contributed equally to this work.

${ }^{1}$ Bristol Centre for Surgical Research, Population Health Sciences, Bristol

Medical School, Room 2.05, Canynge Hall, 39 Whatley Road, Clifton, Bristol

BS8 2PS, UK

Full list of author information is available at the end of the article
}

(c) The Author(s). 2020 Open Access This article is distributed under the terms of the Creative Commons Attribution 4.0 International License (http://creativecommons.org/licenses/by/4.0/), which permits unrestricted use, distribution, and reproduction in any medium, provided you give appropriate credit to the original author(s) and the source, provide a link to the Creative Commons license, and indicate if changes were made. 


\section{Background}

Accurate information regarding the cost-effectiveness of existing and novel surgical interventions is vital to inform policy and ensure scarce resources are used to optimal benefit. Central to the meaningful assessment of cost-effectiveness is the accurate evaluation of resource use [1]. This is commonly undertaken using a 'top-down' or 'gross-costing' approach whereby average costs are used to estimate resource use. For instance, in England, national reference costs based on healthcare resource groups (HRGs) can be used to estimate the average cost per inpatient episode for groups of surgical procedures (e.g. "very major hip procedures for non-trauma") [2]. While this method is straightforward, it is too crude for many purposes. For example, it is not possible to compare the costs of two different surgical procedures within the same procedure group (e.g. cemented vs hybrid total hip replacements) or evaluate a modification to an existing procedure when the procedure itself is unchanged (e.g. single port vs standard multiple port laparoscopic appendicectomy). Furthermore, gross costing approaches may not be suitable for evaluating novel surgical interventions (e.g. robotic cardiac surgery) which do not fall within existing HRGs. Microcosting is a method that allows for more precise assessment of the economic costs of a healthcare intervention [3]. Defined as the 'direct enumeration and costing of every input consumed in the treatment of a particular patient' [4], micro-costing attempts to measure costs of a service as accurately as possible. The process has three stages; the identification of all resources involved in the provision of care (e.g. human-resources/ theatre-time/consumables); accurate measurement of each resource (for example using time-and-motion studies); and valuation of the resources used. Although this approach is time-consuming, it may more accurately reflect the cost of surgical procedures, especially if the procedure is new or includes the use of expensive implants or consumables. It may therefore be the most appropriate method of resource use assessment alongside surgical trials.

The potential value of this methodology has already been identified and a framework has been developed for applying micro-costing methodology to cost evaluations of surgical technologies [5]. This framework focuses specifically on direct costs which reflect the price of resources directly attributable to the procedure rather than indirect costs (e.g. overheads) which have to be estimated using an allocation formula [5]. The framework divides the direct costs into two categories; fixed costs which do not vary according to the level of activity (e.g. personnel costs and medical devices e.g. robotic systems) and variable costs such as reusable instruments and disposables which vary according to the type or number of procedures performed. A recent review of use of micro-costing in bariatric surgery suggests that this framework may be more widely applicable to surgical interventions [6], but further work is required to determine whether it is generalisable to a range of surgical procedures.

Despite the potential benefits of micro-costing, it is time and resource intensive and this may limit its utility in clinical trials. Work is therefore needed to explore whether a more targeted approach may be possible. It is hypothesised that surgical interventions will have 'costdrivers' which create or drive the majority of the cost of the activity. If these are consistent across surgical interventions or can be easily identified, it may be possible to develop a simplified micro-costing framework which could be used in future surgical trials.

The aim of this systematic review was therefore to identify and critically appraise published studies reporting the use of micro-costing in economic analyses of surgical interventions to explore the resources included in each study; the methods used for measuring and valuing resource use and reported cost-drivers of surgical interventions to inform recommendations for the future use of micro-costing in surgical interventions.

\section{Methods}

The protocol for this systematic review was registered on the PROSPERO International prospective register of systematic reviews (reference number CRD42018099604) before data extraction commenced.

\section{Literature search strategy}

The OVID SP versions of MEDLINE, Embase, EconLit, the Cochrane Database and the NHS Economic Evaluation Database (NHS EED) were searched using published micro-costing search strategies [6-8] and appropriate search terms for 'surgery' developed in collaboration with an information specialist (Additional file 1). The combined search strategy was tested iteratively to ensure the sensitivity in identifying micro-costing studies known to the authors.

The search was limited to human studies, published in English from database inception up to and including 22nd June 2018. Abstracts and conference reports were excluded due to difficulty assessing incomplete information.

Duplicate records were excluded, and titles and abstracts of remaining citations were screened for eligibility using prespecified inclusion criteria (see below). The reference lists of identified studies and existing reviews were also manually searched to identify any additional relevant papers. 


\section{Selection of papers}

Full papers reporting the use of micro-costing as a method of resource-use assessment in economic analyses of surgical interventions were eligible for inclusion.

For the purposes of the review, a surgical intervention was defined as any 'procedure involving an incision with instruments usually performed in an operating theatre and normally involving anesthesia and/or respiratory assistance' [9]. Micro-costing was defined as the 'direct enumeration and costing of every input consumed in the treatment of a particular patient' [4]. Initial scoping demonstrated that many studies stated having undertaken a micro-costing exercise but did not report any details of the resources included or how these resources had been measured or valued. Such studies were not considered informative so only those considered to represent a 'sufficiently-detailed micro-costing exercise' were eligible for inclusion.

The definition of a 'sufficiently detailed' micro-costing study was iteratively modified during the pilot abstract screening and data extraction phase which included approximately 100 abstracts and 10 papers respectively. The piloting and iterative modification of the abstract screening and data extraction pro-formas were completed by 2 reviewers (CD/SP) following discussion with experienced health economists (WH/CR). For the purposes of this review, a 'sufficiently-detailed microcosting study' was agreed to be one in which i) the elements of the surgical procedure were sufficiently disaggregated and at the very least two separate elements of the procedure (e.g surgeon costs and consumables) costs presented separately AND ii) the unit cost of at least one element of the procedure (e.g. cost per minute of surgeon time; cost per implant) had been considered. Any studies not meeting both of these criteria were excluded.

Economic analyses using 'top down' or gross-costing approaches (e.g. HRGs), economic models, conceptual papers, reviews, commentaries, letters, abstracts, editorials and studies not evaluating surgery were excluded. Studies comparing surgery with a non-surgical comparator were included provided that micro-costing of the surgical intervention had been performed and met the inclusion criteria.

All papers were screened for inclusion by two reviewers (CD and SP/GD) using a standardised two-stage screening proforma (Additional file 2). Phase 1 (abstract screening) focused on identifying original full papers reporting economic analyses of surgical interventions using a micro-costing approach. Papers meeting these inclusion criteria or in which there was uncertainty proceeded to phase 2 (full-text screening). Only papers meeting all the inclusion criteria following full-text screening were included in the review. Uncertainties that remained after full-text review were resolved by discussion with an experienced health economist (CR/WH). Reasons for exclusion were recorded.

\section{Data extraction}

Data were extracted using a standardised REDCap [10] data extraction proforma and included three sections; i) study and surgical procedure characteristics, ii) critical appraisal and iii) details of the micro-costing methodology reported (Additional file 3).

\section{Study and surgical procedure characteristics}

Study details included year of publication and country of origin; study design (randomised controlled trial or observational study with or without comparison group); prospective or retrospective accrual of data; authorreported type of economic analysis undertaken (e.g. micro-costing; cost analysis, cost-effectiveness analysis; economic analysis; other, irrespective of whether this was considered appropriate); number of participating centres (in the micro-costing study separately); surgical specialty with details of the intervention(s) assessed; whether implants were used (yes or no); type of hospital stay (day-case; inpatient, both or not stated); type of anaesthetic (general, local, both or not stated); details of the study population and stated aims and objectives of the study which were extracted verbatim.

\section{Critical appraisal}

In the absence of a specific quality assessment tool for micro-costing studies, a modified version of the Consensus on Health Economic Criteria (CHEC) Checklist [11] was used to assess the quality of included studies. The CHEC checklist represents a generic core set of 19 items that can be used to assess the methodological quality of economic evaluations. As this review focuses specifically on the use of micro-costing in economic analyses of surgical interventions, items relating to the appropriateness of the selected time horizon (item 5); the quality of outcome assessment (items 10-12) and the appropriate discounting of future costs and outcomes (item 14) were not considered relevant and were not assessed.

Items 7-9 which assess the degree to which all relevant costs were appropriately identified, measured and valued were the focus of the review and were expanded to include additional details (see below).

\section{Reporting of micro-costing methodology}

Details of the micro-costing undertaken in each included study were extracted. This included assessing whether the authors reported performing a 'micro-costing' study or if alternative terms were used; the purpose of the study (e.g. to compare surgical procedure costs or to compare costing methodologies); methods of resource 
identification (e.g. patient pathway mapping, interviews with medical staff,) and the scope of the costing exercise (e.g. pre-operative planning; surgical procedure; postoperative hospital stay).

Resources included in each micro-costing study were recorded (yes/no) using the categories proposed in the existing framework [5] expanded based on initial scoping work and expertise within the study team. These included costs relating to personnel; materials/ disposables; medical devices; reusable instruments; the operating room; inpatient hospital stay; overhead/administration; drugs/medications; investigations/imaging; complications; follow-up and other costs. For each resource category, specific details of what resources were included (e.g. surgeon, nursing and anaesthetist time in 'personnel costs'); and details of how these resources were i) measured (e.g. interviews; time and motion studies) and ii) valued (e.g. invoice amounts; hospital human resources departments; provider price catalogues) were extracted verbatim. Finally, we recorded any cost drivers identified by the authors (yes/no) with verbatim details of cost-drivers reported. Most studies did not report results in sufficient detail for us to apply a quantitative definition to identify 'cost drivers' or compare their relative importance between studies. Therefore, we are limited to providing a narrative summary of author-identified cost drivers. Similarly, there was insufficient detail to determine whether the reported studies distinguished adequately between static cost drivers, accounting for the highest proportion of total cost at a given point in time, and dynamic cost drivers, making the main contribution to the growth of total costs over time.

Data extraction was performed by one reviewer (CD) with a proportion (10\%) checked by a second reviewer (SP/GD). Discrepancies were resolved by discussion with an experienced health economist (CR/ WH).

\section{Analysis}

Descriptive statistics were used to summarise study details; compliance with items in the CHEC checklist and details of the micro-costing reported in each study. Simple content analysis [12] was used to categorise verbatim data relating to study aims and objectives; methods used for identifying, measuring and valuing resources and details of any reported cost-drivers. Stata/MP 15 was used for all quantitative analyses.

\section{Results}

Study selection

Of the 1009 abstracts identified from the electronic searches, 243 full papers were obtained for further evaluation. Of these, 79 met the inclusion criteria and were retained. A further six papers were identified from the hand-search. A total of 85 papers were therefore included in the review (Fig. 1). Included papers are listed in Additional file 4.

\section{Study and procedure characteristics}

The characteristics of the included studies are summarised in Table 1 . Studies were most frequently published between 2016 and $2018(n=34,40.0 \%)$ and the majority originated from Europe $(n=41,48.2 \%)$ or North America $(n=21,24.7 \%)$. Included studies were commonly single-centre $(n=66,77.6 \%)$, retrospective $(n=40,47.1 \%)$, observational studies with $(n=46$, $54.1 \%)$ or without $(n=21,24.7 \%)$ a comparison group. Authors' most frequently described their studies as being 'cost analyses' ( $n=54,63.5 \%)$ and the median number of procedures micro-costed per study was 100 (interquartile range 24-233) although this ranged widely (Table 1).

Orthopaedic $(n=22,25.9 \%)$, general $(n=14,16.5 \%)$ and plastic surgical $(n=9,10.6 \%)$ procedures were micro-costed most frequently but a comprehensive range of surgical specialities were represented and almost a third of studies micro-costed a surgical procedure involving an implant $(n=25,29.4 \%)$. Most procedures were performed under general anesthesia $(n=39,46.4 \%)$ and required an inpatient hospital stay $(n=43,50.6 \%)$ but this information was often not clearly reported (Table 1).

\section{Critical appraisal}

The majority of studies scored highly according to the CHEC checklist (Table 2). Included studies largely had a well-designed research question $(n=85,100 \%)$ in a clearly defined population $(n=84,98.8 \%)$ and were using an appropriate economic study design to achieve their stated objectives $(n=85,100 \%)$. The study perspective was less well-reported $(n=49,57.6 \%)$ and only a quarter of studies $(n=23,27.1 \%)$ performed any form of sensitivity analysis. Most studies discussed the generalisability of the results to other settings and patient/client groups $(n=76,89.4 \%)$. A quarter of studies $(n=21,24.7 \%)$ did not report any conflicts of interest; only half $(n=43$, $50.6 \%$ ) reported obtaining ethical approval for the project.

\section{Reporting of micro-costing methodology}

Table 3 summarises details of the micro-costing undertaken in the included studies. There was a lack of consistency regarding study terminology with just over half of authors $(n=46,54.1 \%)$ describing their methodology as 'micro-costing' while a third $(n=27 / 85,31.8 \%)$ used the term (time-driven) activity-based costing (ABC) and a smaller number referred to using a 'bottom-up' approach $(n=4 / 85,4.7 \%)$. 


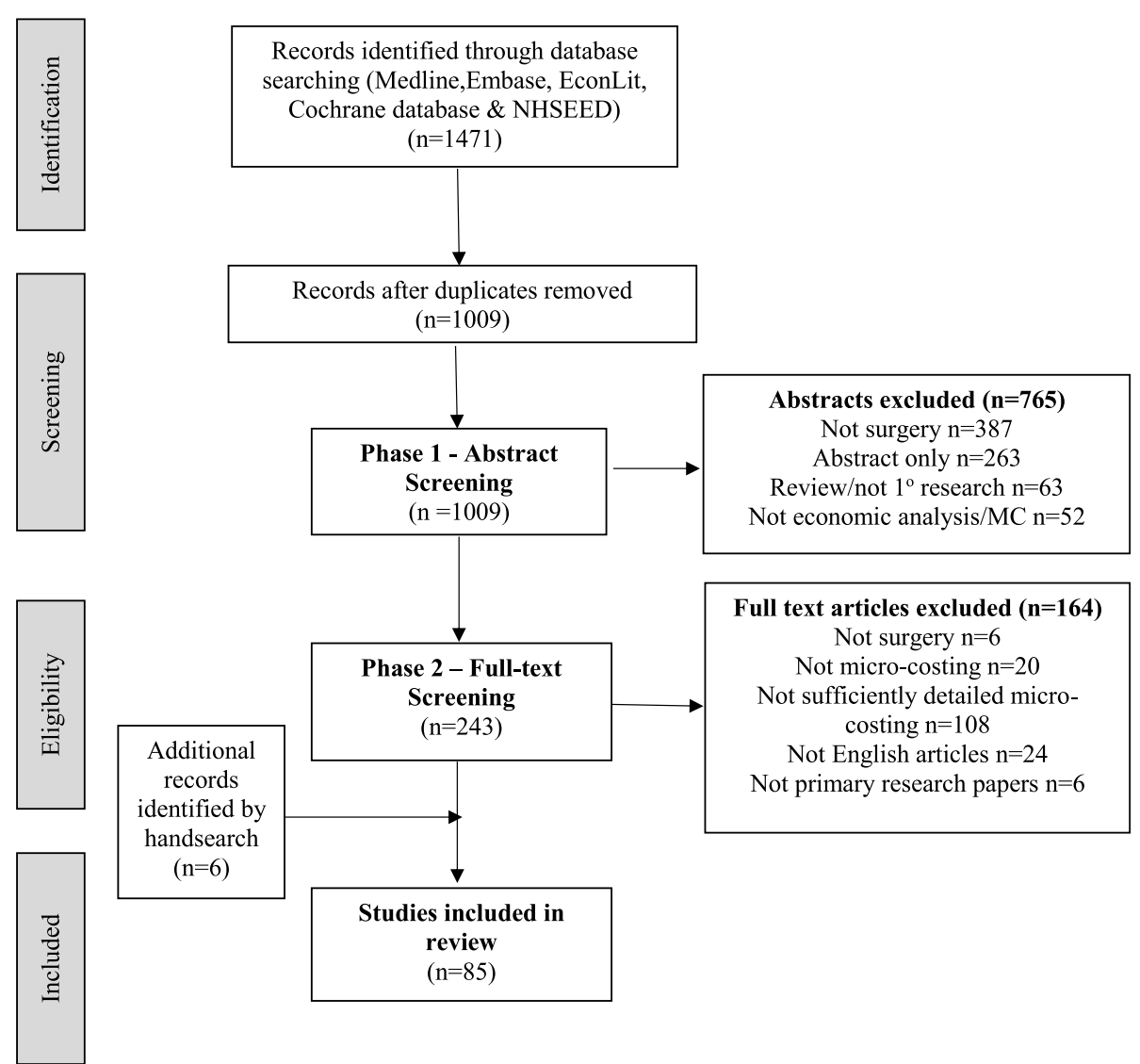

Fig. 1 PRISMA diagram for the systematic review

Micro-costing was most commonly performed to compare procedures, techniques or processes $(n=43,50.6 \%)$ but over a third of studies $(n=29,34.1 \%)$ were specifically undertaken to identify cost-drivers for surgical procedures and a quarter $(n=20,23.5 \%)$ were performed to compare costing methodologies; most commonly gross or 'top-down' methods, such as HRGs or insurance reimbursement with micro-costing or 'bottom-up' approaches. In addition to micro-costing the actual surgical procedure, most studies $(n=72,84.7 \%)$ also micro-costed the patients' hospital stay. Pre-operative investigations required prior to surgery were included in over half the studies $(n=48,56.5 \%)$ and a third microcosted surgical complications $(n=29,34.1 \%)$ and/or follow-up required after discharge $(n=29,34.1 \%)$. Most studies reported including either direct costs only $(n=$ $24,28.2 \%)$ or both direct and indirect costs $(n=29$, $34.1 \%)$ but over a third $(n=32,37.6 \%)$ did not state what types of costs had been considered.

Although most studies included personnel costs $(n=$ $76,89.4 \%)$; materials and disposables $(n=76,89.4 \%)$; operating room costs $(n=62,72.9 \%)$ and/or the costs of any drugs or medications $(n=63,74.1 \%)$ in their microcosting, there was no single type of resource included in all studies and categorisation of resources into predefined categories was often difficult due to different studies aggregating and reporting resources in different ways. There was also a lack of consistency in the ways in which relevant resources were identified, measured and valued. Hospital information systems or administrative databases were used in over $40 \%(n=37,43.5 \%)$ of studies with approximately a third $(n=32,37.6 \%)$ using patient pathway mapping to identify resource-use. Interviews with surgeons and/or patients $(n=26,30.5 \%)$ and direct observation $(n=20,23.5 \%)$ were less commonly used (Table 3).

\section{Identification of cost-drivers}

The majority of included studies $(n=72,84.7 \%)$ reported having identified cost-drivers but these differed according to whether the authors had micro-costed the surgical procedure alone or the whole episode of care (surgical procedure including the inpatient stay $+/$ - preoperative investigations $+/$ - follow-up) (Table 4). For studies just considering the surgical procedure, the main costdrivers were identified as the costs of the operating theatre $(n=17,28.3 \%)$; theatre personnel $(n=15,25.0 \%)$; operative equipment $(n=9,15.0 \%)$, implants $(n=7$, 
Table 1 Study and surgical procedure characteristics $(n=85)$

\begin{tabular}{ll}
\hline & $n(\%)$ \\
\hline Year of publication & $4(4.7)$ \\
Pre 2000 & $5(5.9)$ \\
$2001-2005$ & $11(12.9)$ \\
$2006-2010$ & $31(36.5)$ \\
$2011-2015$ & $34(40.0)$ \\
$2016-2018$ & \\
Study design & $5(5.9)$ \\
Within/as part of RCT & $42(49.4)$ \\
Observational comparative study & $4(4.7)$ \\
Case/control study & $21(24.7)$ \\
Case-series & $13(15.3)$ \\
Other & \\
Data collection & $27(31.8)$ \\
Prospective & $40(47.1)$ \\
Retrospective & $4(4.7)$ \\
Combination of prospective and retrospective & $14(16.5)$ \\
Not clear/not stated &
\end{tabular}

Type of economic analysis reported by study authors

\begin{tabular}{|c|c|}
\hline Cost analysis & $54(63.5)$ \\
\hline Micro-costing/activity-based costing & $13(15.3)$ \\
\hline Cost-effectiveness analysis & $6(7.1)$ \\
\hline Economic analysis & $4(4.7)$ \\
\hline Cost Consequence Analysis & $3(3.5)$ \\
\hline Cost-utility analysis & $1(1.2)$ \\
\hline Other & $4(4.7)$ \\
\hline \multicolumn{2}{|l|}{ Country of origin } \\
\hline Europe & $41(48.2)$ \\
\hline USA & $21(24.7)$ \\
\hline UK & $7(8.2)$ \\
\hline Canada & $4(4.7)$ \\
\hline Other & $6(7.1)$ \\
\hline Multinational & $6(7.1)$ \\
\hline \multicolumn{2}{|l|}{ Number of participating centres } \\
\hline Single centre & $66(77.6)$ \\
\hline Multicentre & $16(18.8)$ \\
\hline Not clear/not stated & $3(3.5)$ \\
\hline $\begin{array}{l}\text { Number of patients/procedures } \\
\text { micro-costed (median, interquartile } \\
\text { range, range) }\end{array}$ & $100(24-233)(6-2130)$ \\
\hline \multicolumn{2}{|l|}{ Surgical speciality } \\
\hline Orthopaedics & $22(25.9)$ \\
\hline General surgery & $14(16.5)$ \\
\hline Plastic surgery & $9(10.6)$ \\
\hline Obstetrics and gynaecology & $8(9.4)$ \\
\hline ENT & $7(8.2)$ \\
\hline
\end{tabular}

Table 1 Study and surgical procedure characteristics $(n=85)$ (Continued)

\begin{tabular}{ll}
\hline & $n(\%)$ \\
\hline Maxillofacial surgery & $5(5.9)$ \\
Cardiothoracic surgery & $5(5.9)$ \\
Urology & $3(3.5)$ \\
Neurosurgery & $2(2.4)$ \\
Vascular surgery & $1(1.2)$ \\
Other & $4(4.7)$ \\
Surgical procedure involving an implant & $25(29.4)$ \\
Type of anaesthesia used & \\
General anaesthesia only & $39(46.4)$ \\
Local anaesthesia only & $4(4.8)$ \\
Both local and general anaesthesia & $5(6.0)$ \\
Not stated/not clear & $36(42.9)$ \\
Type of hospital stay & $13(15.3)$ \\
Day-case procedures & $43(50.6)$ \\
Inpatient procedures & $11(12.9)$ \\
Both day-case and inpatient procedures & $18(21.2)$ \\
Not stated/not clear & \\
\hline
\end{tabular}

$11.7 \%)$ and theatre consumables $(n=6,10.0 \%)$. For studies micro-costing the full episode of care, the main costdrivers identified were the inpatient stay $(n=25,35.7 \%)$ and personnel/labour costs $(n=14,20 \%)$. Use of intensive care $(n=7,10 \%)$; consumables $(n=6,8.6 \%)$ and overheads $(n=6,8.6 \%)$ were also identified as potential cost-drivers in these studies (Table 4).

\section{Discussion}

Micro-costing may offer a more accurate method for costing in economic analyses of surgical interventions, but this review suggests that current use of the methodology is inconsistent and, in many instances, lacking in methodological rigor. There is a lack of consistency regarding terminology used; the types of resources included and the methods by which these are measured and valued. Almost half of studies used retrospectively collected data (e.g. from hospital administrative or information systems). Although routinely available data sources may be accurate, especially in modern 'fee-forservice' settings, other reported methods for resource use identification such as case-note review or operating room logs may be less robust [13-15], potentially compromising the validity of the micro-costing approach. Over three quarters of all studies were undertaken at a single centre limiting the generalizability of the results. Almost half of the included studies did not report resource utilization and unit cost data separately. Instead summary costs were presented with limited disaggregation of individual costs or transparency regarding 
Table 2 Quality assessment of included studies

\begin{tabular}{|c|c|}
\hline & $n(\%)$ \\
\hline \multicolumn{2}{|l|}{ Is the study population clearly defined? } \\
\hline Yes & $84(98.8)$ \\
\hline No & $1(1.2)$ \\
\hline \multicolumn{2}{|c|}{ Are the competing alternatives clearly described? } \\
\hline Yes & $63(74.1)$ \\
\hline Not applicable & $22(25.9)$ \\
\hline \multicolumn{2}{|c|}{ Is a well-designed research question posed in an answerable form? } \\
\hline Yes & $85(100.0)$ \\
\hline \multicolumn{2}{|c|}{ Is the economic study design appropriate to the stated objective? } \\
\hline Yes & $85(100)$ \\
\hline \multicolumn{2}{|c|}{ Is the actual perspective chosen appropriate? } \\
\hline Yes & $48(56.5)$ \\
\hline No & $1(1.2)$ \\
\hline Not stated & $36(42.4)$ \\
\hline \multicolumn{2}{|l|}{ Sensitivity analysis performed } \\
\hline No sensitivity analysis performed & $61(71.8)$ \\
\hline Deterministic sensitivity analysis & $18(21.2)$ \\
\hline Stochastic sensitivity analysis & $2(2.4)$ \\
\hline Other stated sensitivity analysis & $3(3.5)$ \\
\hline Not stated/not clear & $1(1.2)$ \\
\hline \multicolumn{2}{|c|}{ Do the conclusions follow from the data reported? } \\
\hline Yes & $85(100)$ \\
\hline \multicolumn{2}{|c|}{$\begin{array}{l}\text { Does the study discuss the generalizability of the results to other } \\
\text { settings and patient/client groups? }\end{array}$} \\
\hline Yes & $76(89.4)$ \\
\hline No & 9 (10.6) \\
\hline \multicolumn{2}{|c|}{ Do the authors reports any conflict of interest? } \\
\hline Yes & $7(8.2)$ \\
\hline No & $57(67.1)$ \\
\hline Not stated & $21(24.7)$ \\
\hline \multicolumn{2}{|c|}{ Was ethical approval obtained for the study? } \\
\hline Yes & $43(50.6)$ \\
\hline
\end{tabular}

precisely which resources had been included making meaningful interpretation difficult. Some good examples of micro-costing surgical interventions, however, were identified $[16,17]$ and have subsequently been published [18].

There was significant variation in which aspects of the patient pathway researchers elected to micro-cost. Most studies costed both the surgical procedure and the associated inpatient stay, but others also included preoperative planning/investigations, complications and follow-up required following discharge. To an extent, this variation may appropriately reflect the diversity in the aims of micro-costing studies. For economic evaluations comparing two different surgical interventions researchers may legitimately focus on the incremental cost differences between the two procedures and exclude costs that are common to both procedures (e.g. preoperative visits). Furthermore, for economic evaluation, methodological guidelines emphasise that the level of resource use detail required is lower for items with a small relative impact on total costs (e.g. volatile anaesthetic agents) [19]. In contrast, for studies aiming to compare the actual costs of surgery to "top down" costs (e.g. hospital reimbursement), it is important to include all elements contributing to cost. The level of detail may also be constrained by study setting and in particular the granularity of the unit costs available for valuing resources. Studies in settings with itemised fee for service hospital reimbursement have greater scope to cost resource use in much finer detail. Approximately one in four studies compared a measured cost of performing a procedure, as determined by micro-costing, with procedure reimbursement rates, which were often considered to be insufficient. Many of these studies were undertaken by clinicians. The scope of micro-costing (e.g. procedure based or full admission) and the economic perspective (hospital or wider health service) in such studies should be rigorously appraised to ensure it is not biased by clinicians' financial interests. Cost-drivers were identified and reported in most studies, but these differed according to which aspects of the patient pathway were included. There is a need for a standardized definition of a cost driver in the literature so that authors are consistent with this terminology and how it is measured and reported. Although, it was clear from the reported studies in this review that the cost drivers were the resources that involved the highest proportion of the total costs of the surgical pathway and/or surgical procedure, this proportion may in some studies have been below $50 \%$ of total cost and/or may have subsumed several cost drivers instead of only one cost driver per pathway or procedure.

This review has demonstrated the need for specific guidance for researchers undertaking micro-costing. Work to develop a checklist for the conduct, reporting and appraisal of micro-costing studies in healthcare is ongoing [20]. This checklist may improve the quality of future studies but is not specific to surgery which presents unique challenges. A framework for costing surgical technologies [5] provides formulae for estimating the fixed (device and personnel) and variable (re-usable equipment and disposables) costs of surgery. However, the framework only considers the costs of the operative procedure which limits its applicability for trials of surgical procedures likely to have resource-use implications beyond the operating theatre such as differential length of inpatient stay; use of intensive care or need for follow-up investigations. The framework also excludes indirect costs (overheads) of surgery which are necessary 
Table 3 Reporting of micro-costing methodology

Identified as authors as being a micro-costing study?
Yes
No
If no, authors' description of their methodology ( $n=39$ )
Activity-based costing or time driven activity-based
costing
Bottom up approach
Direct/detailed cost calculation
Unit costs
Cost analysis
Other/Unclear
Stated aim of the microcosting study ${ }^{\text {a }}$
To compare procedures/techniques/processes
To determine accurate costs/identify cost drivers
To compare costing methodologies (e.g. micro-costing
and HRGs)
Other stated
What aspects of the patient pathway were micro-costed
Pre-operative planning/investigations
Surgical procedure
Hospital stay
Yomplications of surgery
Rehabilitation (physiotherapy/occupational therapy)

Did the authors report both direct and indirect costs

Direct costs only

Both direct and indirect costs

Not stated

Methods by which resources were identified ${ }^{b}$

Patient pathway mapping

Interviews with surgeons/patients

Accounting/finance department

Hospital information systems/administrative databases

Direct observation

Review of patient notes/charts

Review of operating logs/books

Standardised reporting template

Manufacturer

Case report forms

Other

Table 3 Reporting of micro-costing methodology (Continued)

$n(\%)$

$$
46(54.1)
$$

$39(45.9)$

27 (69.2)

$4(10.3)$

$3(7.7)$

2 (5.1)

1 (2.6)

2 (5.1)

$43(50.6)$

$29(34.1)$

$20(23.5)$

2 (2.3)

$48(56.5)$

$85(100.0)$

$72(84.7)$

29 (34.1)

29 (34.1)

6 (7.1)

1 (1.2)

$50(58.8)$

$35(41.2)$

24 (28.2)

$29(34.1)$

$32(37.6)$

32 (37.6)

$26(30.5)$

17 (20)

$37(43.5)$

$20(23.5)$

$16(18.8)$

5 (5.9)

2 (2.3)

2 (2.3)

$1(1.2)$

$11(12.9)$

$n(\%)$

Resources identified and reported ${ }^{c}$

Personnel costs

$76(89.4)$

Materials/disposables

76 (89.4)

Medical device costs

$34(40.0)$

Re-usable instrument costs

$16(18.8)$

Operating room costs (separate from admission

$62(72.9)$

costs)

Inpatient hospital stay costs

$52(61.1)$

Overhead/administration costs

$46(54.1)$

Medicinal/Drug costs

$63(74.1)$

Imaging/investigation/blood tests costs

39 (45.8)

Complications

$5(5.8)$

Outpatient/Follow up

$9(10.5)$

Perioperative care (pre-operative care/recovery)

$4(4.7)$

Other costs

$10(11.7)$

Did the authors identify cost drivers

Yes

No

$13(15.3)$

HRG healthcare resource groups

${ }^{\text {a }}$ Some studies had more than one stated micro-costing aim

${ }^{\mathrm{b}}$ Most studies report more than one method

${ }^{c}$ Most studies report more than one resource

for micro-costing analyses wishing to compare surgical care with non-surgical care or compare the cost of surgery with procedure reimbursement values. Further work is therefore needed to develop more simplified recommendations for the use of micro-costing in surgery generally and specifically for efficient and effective use of the methodology in surgical trials. Recommendations based on the findings of this review are summarised in Table 5.

This is, to our knowledge, the first study to systematically identify and critically-appraise the use of microcosting as a method of resource-use assessment in surgery. We used published search strategies for microcosting $[6,7]$, however due to the lack of standardisation in micro-costing terminology and excluding non-English language papers, our search may have overlooked other relevant studies. The review was restricted to studies meeting our prespecified definition of being a 'sufficiently-detailed micro-costing exercise'. This led to the exclusion of a large number of studies at the full-text screening stage. Inclusion of these studies would not have been informative, but it is important to note that costing studies in surgery more generally may be methodologically less robust than those included in this review. The majority of included studies scored highly on the CHEC checklist despite an objective lack of methodological rigor. This highlights the need for specific 
Table 4 Main cost drivers identified by included studies ${ }^{a}$ $(n=72)$

\begin{tabular}{|c|c|c|}
\hline Main Cost-drivers & $\begin{array}{l}\text { Cost drivers within } \\
\text { the surgical } \\
\text { procedure alone } \\
(n, \%)\end{array}$ & $\begin{array}{l}\text { Cost drivers within } \\
\text { the full episode } \\
\text { of care } \\
(n, \%)\end{array}$ \\
\hline $\begin{array}{l}\text { Operating room/theatre } \\
\text { cost (surgery cost/operating } \\
\text { room time) }\end{array}$ & $17(28.3)$ & $0(0.0)$ \\
\hline Personnel/labour costs & $15(25.0)$ & $14(20.0)$ \\
\hline $\begin{array}{l}\text { Operative equipment } \\
\text { (disposable and non- } \\
\text { disposable, including robots) }\end{array}$ & $9(15.0)$ & $0(0.0)$ \\
\hline $\begin{array}{l}\text { Implant/device used in } \\
\text { procedure }\end{array}$ & $7(11.7)$ & $0(0.0)$ \\
\hline Consumables & $6(10.0)$ & $6(8.6)$ \\
\hline Medications (including blood) & $4(6.6)$ & $5(7.0)$ \\
\hline Inpatient stay & NA & $25(35.7)$ \\
\hline Intensive care & NA & $7(10.0)$ \\
\hline $\begin{array}{l}\text { Physiotherapy and } \\
\text { rehabilitation }\end{array}$ & NA & $3(4.3)$ \\
\hline Overheads & $0(0.0)$ & $6(8.6)$ \\
\hline Complications & $2(3.3)$ & 0 \\
\hline Pre-operative visits & NA & $1(1.4)$ \\
\hline Medical aids & NA & $1(1.4)$ \\
\hline Imaging and diagnostic tests & NA & $1(1.4)$ \\
\hline Other & $0(0.0)$ & $1(1.4)$ \\
\hline
\end{tabular}

${ }^{a}$ Main cost drivers reported by authors. Other cost driver were identified but these were stated as the 'main' or most important ones

recommendations for the design, conduct and critical appraisal of micro-costing studies to improve the quality and value of this work.

Micro-costing produces an accurate assessment of resource-use but the methodology is time consuming and

Table 5 Recommendations for the efficient use of microcosting as a method of resource-use assessment in surgery

\begin{tabular}{l} 
Key Recommendations \\
\hline 1. Consistently use the term 'micro-costing' when describing the \\
methodology and include 'micro-costing' in the abstract and as a \\
keyword to facilitate future identification of studies \\
2. Identify the potential key cost-drivers (see Table 4) for the surgical \\
intervention based on the research question. Patient pathway map- \\
ping with experts (e.g. surgeons and other healthcare professionals) \\
may help identify key resources. \\
3. For comparative cost analyses, more accurate albeit time \\
consuming methods (e.g observation) are warranted for resources \\
that differ between comparator procedures, whereas cruder \\
methods (e.g expert opinion) may be sufficient for inexpensive \\
resources with similar use between procedures. \\
4. Ensure transparent reporting of micro-costing studies with sufficient \\
disaggregation of elements of the procedure/pathway and report- \\
ing of unit costs (in supplementary material if necessary) \\
5. Consider applying focused cost-driver micro-costing at multiple \\
centres to improve generalisability of the results
\end{tabular}

resource intensive and universal application alongside surgical trials would not be practical $[3,21]$. A more targeted or hybrid micro-costing approach, however, may have value [22]. Almost all studies included in the review identified one or more 'cost-drivers' that represented the greatest proportion of the costs for their procedures. These differed according to the aspects of the patient pathway considered as for example, operating room costs were the main cost-driver in studies just costing the surgical procedure but were less important in studies costing the full episode of care. Costdrivers may also be influenced by other factors such as the specific research question and the intervention under study. For example, a study comparing the cost of two surgical procedures identical except for the implant used might reasonably focus on implant cost and detailed observations of surgeon time spent inserting the implant. Focusing attention on identifying and accurately costing these key elements of care while applying less detailed methods (e.g. record review or expert opinion) for other components may allow the benefits of micro-costing to be realized in an efficient way. Process mapping with experts including surgeons, nurses, other allied healthcare professionals and if appropriate, patients themselves may be an effective way to identify key cost elements. More targeted, efficient micro-costing of key costdrivers may also allow this methodology to be applied over a wider number of centres in a trial setting improving both the accuracy of the costing data obtained and the generalizability of the results [23]. Further work, however, is needed to determine if this targeted approach would be feasible alongside a surgical trial.

As health systems and technologies become more advanced, routinely-available data may have increasing utility for resource-use assessment and may make future large-scale micro-costing studies possible. Healthcare provider cost-accounting systems that capture details on all resources used in an episode of care combined with electronic theatre systems that could provide proxy time and motion data may represent a time and cost-efficient method for detailed resource-use assessment. Such systems and methodology, however, would need to be consistent across centres if the costs generated were to be comparable. This may be challenging, especially across different healthcare settings and geographical locations.

Micro-costing has the potential to improve the accuracy of economic analyses of surgical interventions by providing more accurate assessments of resource-use but the overall quality of existing studies is poor. There is a need to improve the consistency and efficiency of micro-costing in surgery if the potential value of the methodology is to be realised. This review highlights a number of ways in which this could be achieved. Using standardised terminology for micro-costing studies; focusing on identifying and accurately costing cost-drivers relevant to the specific research question and 
transparently reporting disaggregated costs for each included resource may represent a simple strategy for improving the design and delivery of future studies.

\section{Conclusions}

Micro-costing may provide a more accurate method of resource-use assessment in economic analyses of surgical interventions and could improve the value of economic evaluations conducted alongside surgical trials, but this systematic review suggests that current use of micro-costing in surgery is inconsistent and lacking in methodological rigor. Using standardised terminology for micro-costing studies; focusing on identifying and accurately costing cost-drivers relevant to the specific research question and transparently reporting disaggregated costs for each included resource may represent a simple strategy for the optimal design and delivery of future studies.

\section{Supplementary information}

Supplementary information accompanies this paper at https://doi.org/10. 1186/s13561-020-0260-8.

Additional file 1. Search strategy for Ovid Medline (1946 to present) (search 1 to 21 ref Doble et al 2017).

Additional file 2. Screening log (Phase 1 and 2).

Additional file 3. Data Extraction Form,

Additional file 4 Papers included in the systematic review $(n=85)$.

\section{Abbreviations}

ABC: Activity-based costing; CHEC: Consensus on Health Economic Criteria; HRGs: Healthcare resource groups; NHS EED: NHS Economic Evaluation Database

\section{Acknowledgements}

The authors would like to thank Cath Borwick for her assistance in developing the search strategy.

\section{Research transparency}

The search strategy; abstract screening and data extraction forms are included as additional files to the manuscript. STATA code for the analysis is available on request

\section{Study registration}

This systematic review was registered at the International Prospective Register of Systematic Reviews (PROSPERO) (registration number CRD42018099604) on 06/06/2018. https://www.crd.york.ac.uk/prospero/ display_record.php?RecordID=99604.

\section{Authors' contributions}

SP and WH conceived the study; SP, CD, WH and CR wrote the protocol; $C D$, $\mathrm{GD}$, SP screened abstracts and performed data extraction; WH and CR provided health economic expertise; SP and CD analysed the results; all authors contributed to interpretation of the data; CD wrote the first draft of the paper; all authors critically reviewed and approved the manuscript prior to submission.

\section{Funding}

This work is funded by a National Institute for Health Research Clinician Scientist Award (CS-2016-16-019). The views and opinions expressed are those of the authors and do not necessarily reflect those of the National Institute for Health Research, National Health Service or the Department of Health and Social Care.
The funding source had no role in the study design; data collection, analysis or manuscript preparation. The decision to publish was made by the study team (SP and WH). The authors had full access to the study data that supports the publication.

\section{Availability of data and materials}

Data included in this paper will be made available on reasonable request from the corresponding author when all analyses have been completed.

Ethics approval and consent to participate

Not applicable.

\section{Consent for publication}

Not applicable.

\section{Competing interests}

The authors declare that they have no competing interests.

\section{Author details}

${ }^{1}$ Bristol Centre for Surgical Research, Population Health Sciences, Bristol Medical School, Room 2.05, Canynge Hall, 39 Whatley Road, Clifton, Bristol BS8 2PS, UK. 'Bristol Breast Care Centre, North Bristol NHS Trust, Southmead Road, Bristol BS10 5NB, UK. ${ }^{3}$ Population Health Sciences, Bristol Medical School, University Of Bristol, Southmead Road, Bristol BS8 2PS, UK.

Received: 28 June 2019 Accepted: 15 January 2020

Published online: 29 January 2020

\section{References}

1. Ridyard $\mathrm{CH}$, Hughes DA. Methods for the collection of resource use data within clinical trials: a systematic review of studies funded by the UK health technology assessment program. Value Health. 2010;13(8):867-72.

2. Improvement N. Reference Costs 2019 [Available from: https://improvement. nhs.uk/resources/reference-costs/. Accessed June 2018.

3. Polsky D, Glick H. Costing and cost analysis in randomised trials: caveat emptor. Pharmacoeconomics. 2009;27(3):179-88.

4. Gold M, Siegel J, Russell L, Weinstein M. Cost-effectiveness in health and medicine. New York: Oxford University Press; 1996

5. Ismail I, Wolff S, Gronfier A, Mutter D, Swanstrom LL. A cost evaluation methodology for surgical technologies. Surg Endosc. 2015;29(8):2423-32.

6. Doble B, Wordsworth S, Rogers CA, Welbourn R, Byrne J, Blazeby JM. What are the real procedural costs of bariatric surgery? A systematic literature review of published cost analyses. Obes Surg. 2017;27(8):2179-92.

7. Xu X, Grossetta Nardini HK, Ruger JP. Micro-costing studies in the health and medical literature: protocol for a systematic review. Syst Rev. 2014:3(1):1-7.

8. Cousins S, Blencowe NS, Blazeby JM. What is an invasive procedure? A definition to inform study design, evidence synthesis and research tracking. BMJ Open. 2019;9(7):e028576

9. Blencowe NS, Boddy AP, Harris A, Hanna T, Whiting P, Cook JA, et al. Systematic review of intervention design and delivery in pragmatic and explanatory surgical randomized clinical trials. Br J Surg. 2015;102(9): 1037-47.

10. Harris PA, Taylor R, Thielke R, Payne J, Gonzalez N, Conde JG. Research electronic data capture (REDCap)--a metadata-driven methodology and workflow process for providing translational research informatics support. J Biomed Inform. 2009:42(2):377-81.

11. Evers $\mathrm{S}$, Goossens $\mathrm{M}$, de Vet $\mathrm{H}$, van Tulder M, Ament A. Criteria list for assessment of methodological quality of economic evaluations: consensus on health economic criteria. Int J Technol Assess Health Care. 2005;21(2): 240-5.

12. Hsieh HF, Shannon SE. Three Approaches to Qualitative Content Analysis. Qual Health Res. 2005;15(9):1277-88.

13. Frick KD. Microcosting quantity data collection methods. Med Care. 2009; 47(7 Suppl 1):S76-81.

14. Gyldmark M. A review of cost studies of intensive care units: problems with the cost concept. Crit Care Med. 1995;23(5):964-72.

15. Smith MW, Barnett PG. Direct measurement of health care costs. Med Care Res Rev. 2003;60(3 Suppl):74s-91s. 
16. Filetti S, Ladenson PW, Biffoni M, D'Ambrosio MG, Giacomelli L, Lopatriello S. The true cost of thyroid surgery determined by a micro-costing approach. Endocrine. 2017;55(2):519-29.

17. Chen A, Sabharwal S, Akhtar K, Makaram N, Gupte CM. Time-driven activity based costing of total knee replacement surgery at a London teaching hospital. Knee. 2015;22(6):640-5.

18. Doble B, Welbourn R, Carter N, Byrne J, Rogers CA, Blazeby JM, et al. MultiCentre micro-costing of roux-en-Y gastric bypass, sleeve Gastrectomy and adjustable gastric banding procedures for the treatment of severe, complex obesity. Obes Surg. 2019;29(2):474-84

19. Drummond M, Sculpher, M.J, Torrance, G.W, O'Brien B, Stoddard G. Methods for the Economic Evaluation of Health Care Programmes. Oxford: Oxford Medical Publications; 2005.

20. Ruger JP, Reiff M. A checklist for the conduct, reporting, and appraisal of microcosting studies in health care: protocol development. JMIR Res Protoc. 2016;5(4):e195.

21. Mogyorosy Z, Smith P. The main methodological issues in costing healthcare services. A literature review. York: Centre for Health Economics (CHE) The University of York; 2005.

22. Wilson EC, Mugford M, Barton G, Shepstone L. Efficient research design: using value-of-information analysis to estimate the optimal mix of topdown and bottom-up costing approaches in an economic evaluation alongside a clinical trial. Med Decis Making. 2016;36(3):335-48.

23. Graves N, Walker D, Raine R, Hutchings A, Roberts JA. Cost data for individual patients included in clinical studies: no amount of statistical analysis can compensate for inadequate costing methods. Health Econ. 2002;11(8):735-9.

\section{Publisher's Note}

Springer Nature remains neutral with regard to jurisdictional claims in published maps and institutional affiliations.

Ready to submit your research? Choose BMC and benefit from:

- fast, convenient online submission

- thorough peer review by experienced researchers in your field

- rapid publication on acceptance

- support for research data, including large and complex data types

- gold Open Access which fosters wider collaboration and increased citations

- maximum visibility for your research: over $100 \mathrm{M}$ website views per year

At $\mathrm{BMC}$, research is always in progress.

Learn more biomedcentral.com/submissions 